\title{
Una mirada a los recreos escolares: El sentir y pensar de los niños y niñas
}

\section{An Overview of School Recess: What Children Think and Feel}

\author{
Ana Lucía Chaves Álvarez \\ Instituto de Estudios Interdisciplinarios de la Niñez y la Adolescencia \\ Centro de Investigación y Docencia en Educación \\ Universidad Nacional \\ Heredia, Costa Rica \\ ana.chaves.alvarez@una.cr
}

Recibido 29 de noviembre de 2012 • Corregido 25 de febrero de 2013 • Aceptado 13 de marzo de 2013

Resumen. En el Instituto de Estudios Interdisciplinarios de la Niñez y la Adolescencia (INEINA) surge la preocupación y el interés por investigar y analizar a fondo qué es lo que está ocurriendo en relación con los recreos en las escuelas públicas del país, y qué es lo que niños y niñas escolares requieren para este momento de esparcimiento, todo esto a la luz de las opiniones de esta población infantil. Este artículo sintetiza la investigación realizada con un grupo de niños y niñas en torno a la temática del recreo, con el fin de dar a conocer las percepciones de la población infantil participante y, a la vez plantear, posibles estrategias que contribuyan a mejorar y fortalecer el recreo en la escuela.

Palabras claves. Recreo, tiempo, espacio, niños y niñas, docentes, Revista Electrónica Educare, Costa Rica.

Abstract. Researchers of the Institute for Interdisciplinary Studies of Childhood and Adolescence (INEINA) have been interested in studying the current activities developed during recess time in public schools and what children require for this period from the point of view of the child population. This paper is the result from a research conducted on this matter with the participation of a group of children, in order to collect ideas from the participants and provide recommendations on possible strategies to enhance and strenghten recreation time at schools.

Keywords. Recess, time, space, children, teachers, Educare Electronic Journal, Costa Rica.

1 Mágister en Pedagogía con énfasis en Desarrollo y Atención Integral de la Primera Infancia (UNA). Licenciada en Pedagogía con énfasis en Educación Preescolar (UNA). Experiencia docente en instituciones educativas costarricenses. Actualmente labora como académica de la División de Educación Básica e investigadora del Instituto de Estudios Interdisciplinarios de la Niñez y la Adolescencia, del Centro de Investigación y Docencia en Educación, (CIDE), Universidad Nacional, Heredia, Costa Rica. Ha impartido diferentes cursos de la Carrera de Pedagogía con énfasis en Preescolar y ha trabajado como miembro del equipo del Proyecto de Acreditación de la División de Educación Básica, del CIDE, de la Universidad Nacional, así como en los procesos de Intervención Pedagógica del I Nivel de Diplomado de la Carrera de Pedagogía con énfasis en Educación Preescolar, UNA. 
URL: http://www.una.ac.cr/educare

CORREO: educare@una.cr

... que es una gran bendición los recreos, si no los tuviéramos nos estuviéramos muriendo de sed y calor y hambre.

Estudiante de tercer grado

\section{Introducción}

Pellegrini y Smith, citados por Jarret (2002), definen el recreo como“(...) un rato de descanso para los niños típicamente fuera del edificio" (p. 2). Por su parte Gómez, citado por Pérez y Collazos (2007), manifiesta que el recreo es un "(...) lapso de tiempo en el cual los escolares realizan espontáneamente actividades recreativas por gusto y voluntad propias y que merecen una esmerada atención por parte de los maestros de la institución (...)" (p. 9).

Para los estudiantes escolares, el momento de recreo es un tiempo de esparcimiento y de disfrute, pues implica salirse de la rutina de las clases $y$, de alguna u otra manera, sentirse liberados. De acuerdo con Aguilera (2010): "(...) Todo niño al sentirse libre en un espacio como el recreo manifiesta su conducta de forma espontánea y real (...)" (p. 3).

En el marco de lo anterior, el presente artículo surge de la investigación realizada en el INEINA titulada "Proyecto: Recreos: sentir y pensar de los niños y las niñas". El punto de partida de la investigación consistió en 6 objetivos específicos, que se detallan seguidamente:

- Conocer la opinión de los niños y niñas de escuelas primarias con respecto a los recreos.

- Identificar cuáles son las características de los recreos de las escuelas primarias a partir de la opinión de los niños y niñas.

- Determinar el rol de los adultos en los recreos a partir de la opinión de los niños y niñas de primero y segundo ciclo.

- Identificar las acciones de los niños y niñas durante el recreo.

- Determinar los intereses y necesidades de los niños y las niñas durante el recreo.

- Plantear una propuesta para el Ministerio de Educación Pública, basada en recomendaciones relacionadas con la temática de los recreos, a partir de la indagación teórica realizada y el sentir de los niños y niñas con respecto al recreo. 
A partir de estos objetivos, se hace un recorrido por aspectos teóricos fundamentales relacionados con el tema de los recreos. Posteriormente, se detalla la metodología y los resultados obtenidos en la investigación, para terminar con el planteamiento de recomendaciones enfocadas a propiciar la mejora y el fortalecimiento de estos tiempos de esparcimiento de la jornada escolar.

\section{¿Por qué el recreo es importante?}

Existen diversas definiciones con respecto al concepto de "recreo", las cuales coinciden con el hecho de que es un tiempo en el que los niños y niñas tienen una oportunidad para jugar y descansar del período formal de las clases. Jarret (2002) manifiesta que en “(...) comparación con el resto del día escolar, el recreo es un tiempo en que los niños gozan de más libertad para escoger qué hacer y con quién" (p. 2). Por su parte, Smaliroff (s. f.) argumenta que es el "(...) intervalo entre las horas de clases escolares, es un momento en el cual los alumnos y los docentes se distienden de las actividades aúlicas, buscando un momento de vínculos espontáneos, recreación y relax" (p. 2).

En relación con lo anterior, son varios los autores que se refieren a los beneficios y aspectos positivos de este momento específico de la rutina escolar. Álvarez et al. (2010) anotan que en los momentos de esparcimiento, se puede descansar y relajarse, aspectos que pueden considerarse importantes del recreo. De este modo plantean que:

Toda persona goza de espacio del tiempo libre de las obligaciones cotidianas de estudio o de trabajo, dicho tiempo libre sirve idealmente para el descanso, el alejamiento de la rutina y la realización de las actividades diferentes pero relajantes (...). (p. 2)

Paralelamente, Pérez y Collazos (2007) establecen una comparación entre el patio de recreo y el aula, las cuales son consideradas como ventajas. De esta forma plantean que:

En el aula el niño no puede elegir con quién sentarse, en el patio puede elegir con quién jugar; el aula es un espacio cerrado, el patio es un espacio abierto; en el aula el control es casi total, en el patio es difuso, el recreo significa libertad, el poder ser tal cual se es, es decir el patio de recreo es un espacio para el esparcimiento, la libertad y la diversión, allí no existen restricciones en cambio sí la verdadera expresión del ser. (p. 15) 
URL: http://www.una.ac.cr/educare

CORREO: educare@una.cr

Por su parte, Pica (s. f.) plantea que el recreo no debe negarse a la población infantil y que los beneficios de este abarcan la mayoría de las áreas del desarrollo. En este sentido destaca siete razones por las cuales los niños y niñas necesitan del recreo.

1. Todos se benefician de un descanso. Se dice que tanto la niñez como las personas adultas aprenden mejor y de forma más rápida cuando sus esfuerzos se distribuyen y cuando se tiene un cambio en el ritmo de trabajo. En el caso de los niños pequeños es importante tomar en cuenta que no pueden procesar la información tan eficazmente como los niños más grandes, debido a la inmadurez de su sistema nervioso y su falta de experiencia.

2. El recreo aumenta el tiempo dedicado a la tarea. Diversas investigaciones demostraron que los niños son más eficientes en sus trabajos cuando tienen momentos de descanso y esparcimiento.

3. Los niños y las niñas necesitan la luz exterior. La luz exterior estimula la glándula pineal, la parte del cerebro que ayuda a regular nuestro reloj biológico, es importante para el sistema inmunológico y hace a las personas sentirse mejor.

4. El juego físico no estructurado reduce el estrés. La Asociación Nacional para la Educación de Niños Pequeños (National Association for the Education of Young Children, NAEYC) recomienda el juego físico sin estructura como una forma de desarrollo apropiada para la reducción del estrés en la vida de los niños y niñas. Para muchos infantes, el recreo es una oportunidad para desahogarse; al aire libre los niños y niñas puede tener comportamientos fuertes, desordenados y ruidosos, los cuales, se consideran inapropiados en las aulas.

5. Los niños y las niñas necesitan aprender a ser seres sociales. Se plantea que el recreo puede ser la única vez durante el día cuando los párvulos tienen la oportunidad de experimentar la socialización y la comunicación real. Los barrios y las comunidades ya no son lo que solían ser, por lo que una vez que se termina la jornada escolar, puede haber muy poca oportunidad de tener una interacción social. Por otra parte, las políticas de algunas escuelas se caracterizan por limitar este contacto con los demás, entonces ¿cómo pueden aprender los niños aprender a vivir y compartir juntos?, ¿cómo y cuándo han aprendido?

6. La salud de la niñez está en riesgo. Muchos de los niños y niñas de la actualidad están sufriendo de sobrepeso y obesidad, pero aun los que no tienen estos problemas, necesitan actividad física para tener una salud óptima. Estar al aire libre permite practicar nuevas destrezas físicas, experimentar la alegría del movimiento y quemar calorías. 
7. La actividad física alimenta el cerebro. Las investigaciones revelan que la mayor parte del cerebro se activa durante la actividad física, mucho más que cuando se trabaja sobre el escritorio. Se optimiza el rendimiento del cerebro porque el movimiento aumenta la capacidad de los vasos sanguíneos, permitiendo la entrega de oxígeno, agua y glucosa al cerebro. Además, diversas investigaciones han demostrado que los estudiantes más activos físicamente han mejorado el rendimiento académico, logran mayores resultados en las pruebas y demuestran una mejor actitud hacia la escuela.

En síntesis, una vez revisadas las diversas fuentes bibliográficas, cabe afirmar que el recreo puede concebirse como un espacio que permite el desarrollo integral de los niños y las niñas, pues no solamente implica el movimiento y la actividad física, sino que contribuye al desarrollo del lenguaje emocional, cognitivo y social. Todas estas bondades deben ser tomadas en cuenta por el personal docente, con el fin de propiciar espacios de recreo sanos, seguros y adecuados para la población infantil.

\section{Un breve marco legal en relación con los recreos}

Jarret y Waite-Stupiansky (2009) consideran que el recreo es un derecho y no un privilegio. A su vez destacan que privar a un infante del recreo a manera de castigo es similar a privar a un niño de su almuerzo, ya que no únicamente es injusto, sino que también inútil. Tomando lo anterior como punto de partida, se considera necesario hacer referencia a diferentes documentos ubicados dentro de un marco legal, en relación con los derechos de la niñez en cuanto a la importancia del juego, el descanso y el tiempo libre, aspectos que están estrechamente vinculados con los recreos.

Así, en la Convención sobre los Derechos del Niño (2005), resaltan dos artículos en los que el juego, el descanso y el desarrollo integral se conciben como derechos de los niños y las niñas y, por lo tanto, a los cuales se puede responder propiciando espacios de recreo durante las jornadas escolares. Estos artículos son los siguientes:

Artículo 27: Los Estados Partes reconocen el derecho de todo niño a un nivel de vida adecuado para su desarrollo físico, mental, espiritual, moral y social. (p. 31)

Artículo 31: Los Estados Partes reconocen el derecho del niño al descanso y el esparcimiento, al juego y a las actividades recreativas propias de su edad y a participar libremente en la vida cultura y en las artes. (p. 36) 
URL: http://www.una.ac.cr/educare

CORREO: educare@una.cr

Sumado a lo anterior, en el Código de la Niñez y la Adolescencia de Costa Rica (1998) se pueden rescatar los siguientes artículos, de los cuales se infiere que el recreo es fundamental, ya que propicia el juego, el desarrollo integral y entre otros aspectos, la recreación. Como se puede ver, los encargados de la población infantil deben asumir la responsabilidad de velar para que estas oportunidades se den y se cumplan.

\section{Artículo 73.- Derechos culturales y recreativos.}

Las personas menores de edad tendrán derecho a jugar y participar en actividades recreativas, deportivas y culturales, que les permitan ocupar provechosamente su tiempo libre y contribuyan a su desarrollo humano integral, con las únicas restricciones que la ley señale. Corresponde en forma prioritaria a los padres, encargados o representantes, darlas las oportunidades para ejercer estos derechos.

El Consejo Nacional de Espectáculos Públicos y las demás autoridades competentes velarán porque las actividades culturales, deportivas, recreativas o de otra naturaleza, sean públicas o privadas que se brinden a esta población estén conformes a su madurez y promuevan su pleno desarrollo. (pp. 73-74)

\section{Artículo 75.- Infraestructura recreativa y cultural}

El Ministerio de Cultura, Juventud y Deportes y las corporaciones municipales establecerán las políticas necesarias y ejecutarán las acciones pertinentes para facilitar, a las personas menores de edad, los espacios adecuados a nivel comunitario y nacional, que les permitan ejercer sus derechos recreativos y culturales.

Los campos deportivos, gimnasios y la infraestructura oficial adecuada para la práctica del deporte o actividades recreativas, estarán a disposición de ese grupo en condicione de plena igualdad, de acuerdo con las reglamentaciones que se emitan. (p. 77)

Por otra parte, Vicente y Castro (2010) hacen referencia a algunos derechos de la niñez, no tradicionales, y entre ellos rescatan el derecho al "(...) juego, entretenimiento, practicar el deporte de su agrado (...), seguridad cuando practica deportes (...), amistad (...), ocio (...), tener adecuadas pautas para descansar (...), tener ambientes agradables, ambientes saludables y limpios" (pp. 89 -90). Todos estos derechos se pueden enmarcar dentro de la importancia del recreo y del juego, ya que, indiscutiblemente, al permitir espacios de recreo se favorece el cumplimiento y respeto de estos derechos. Una vez más se hace un llamado a la reflexión de los adultos para dar a los recreos la importancia que estos tienen. 


\section{Requerimientos que debe contemplar el recreo en la escuela}

La National Association for Sport and Physical Education (2006) propone una serie de requerimientos que deben ser tomados en cuenta en los momentos de recreo en la escuela. A continuación se detallan:

1. Todos los niños y niñas en las escuelas primarias debe participar en al menos un período diario de receso de 20 minutos.

2. El receso no sustituye las clases de Educación Física. Estas proporcionan instrucción secuencial para mejorar el desarrollo de habilidades motoras, conceptos de movimiento y la aptitud física. El recreo permite el juego no estructurado y brinda la posibilidad de participar en la actividad física.

3. El recreo no debe ser visto como una recompensa, sino como un apoyo educativo necesario para todos los niños y niñas. Por tal razón, no se les debe negar el recreo al estudiantado, para que terminen sus trabajos de clase y tampoco como una forma de castigo.

4. Los espacios para el recreo deben ser adecuados y seguros y deben permitir que el estudiantado sea físicamente activo.

5. Los espacios exteriores se deben utilizar cada vez que el tiempo lo permita.

6. Se debe contemplar el equipo adecuado, seguro y apropiado para el desarrollo de los recreos, de manera que le permita a los estudiantes y a las estudiantes participar en la actividad física placentera.

7. El personal docente debe potenciar el desarrollo de habilidades sociales durante el recreo, por ejemplo: cooperación y resolución de conflictos.

8. Las reglas de seguridad deben ser tomadas en cuentas en el momento del recreo.

9. El recreo debe estar correctamente supervisado por adultos calificados.

10. El acoso o comportamiento agresivo no deben ser tolerados en este momento de esparcimiento.

11. Los adultos deben intervenir cuando la seguridad física o emocional de un infante esté en riesgo.

12. El receso no debe estar programado inmediatamente antes o después de la clase de Educación Física.

13. El receso no debe interferir con las clases de Educación Física, que están teniendo lugar en un espacio o entorno en común. 
URL: http://www.una.ac.cr/educare

CORREO: educare@una.cr

\section{Metodología de la investigación}

Esta investigación puede catalogarse como de tipo mixto, ya que se basa en métodos cuantitativos y cualitativos para su desarrollo. Se fundamenta en un enfoque cuantitativo pues, de acuerdo con Hernández, Fernández y Batista (2010), los datos se representan mediante números, y se pretende generalizar los resultados encontrados en una muestra a una población, con miras a las mejoras de los recreos en el sistema educativo público costarricense. De este modo, "(...) las conclusiones derivadas contribuirán a la generación de conocimiento" (Hernández et al., 2010. p. 6).

Sumado a lo anterior, esta investigación también tiene características de un enfoque cualitativo, ya que se utilizó, como técnica para la recolección de datos, la pregunta abierta (Hernández et al., 2010), para que los niños y niñas de diferentes centros educativos dieron su opinión y percepción con respecto a los recreos escolares.

\section{Población participante}

La población participante consiste en los niños y niñas de 5 escuelas públicas costarricenses. La muestra consta de 206 estudiantes de estas escuelas públicas, que cursan desde segundo hasta sexto grado.

\section{Instrumentos utilizados}

Para el desarrollo de la investigación, se utilizó un cuestionario de 17 preguntas abiertas. Este cuestionario se construyó con base en la revisión bibliográfica sobre la temática de recreos, los objetivos propuestos en la investigación, la experiencia de los investigadores y otros aportes de académicos del INEINA.

\section{Análisis de datos}

Dada la naturaleza cualitativa del estudio, los datos de carácter abierto se organizaron en diferentes categorías. Por su parte, debido a la naturaleza cuantitativa de la investigación, se cuantificaron en frecuencias y los porcentajes, se realizaron diferentes tablas para presentar la información y, finalmente, se llevaron a cabo los análisis de la información.

Para la realización de este análisis se realizó un contraste entre la teoría y las opiniones de los niños y niñas participantes y, posteriormente, todo este análisis constituyó la base para la propuesta de recomendaciones que se presenta. 


\section{Principales resultados $\mathbf{y}$ análisis}

Se destacan, a continuación, los datos más relevantes de la información recopilada a partir de las opiniones de los niños y niñas participantes en la investigación.

Como primer elemento importante, se les consultó "¿Qué es lo que más te gusta del recreo?", en la tabla 1 se presentan las respuestas brindadas.

Tabla 1

Lo que más le gusta a los niños y niñas del recreo

\begin{tabular}{lcc}
\hline Categoría & Frecuencia & $\%$ \\
\hline Jugar & 124 & 42,17 \\
Comer & 33 & 11,22 \\
Divertirse & 18 & 6,12 \\
Descansar & 17 & 5,78 \\
Estar con los amigos & 16 & 5,44 \\
Conversar. Hablar & 12 & 4,08 \\
Compartir & 10 & 3,40 \\
Que no hay clases. No hay estudio. & 9 & 3,06 \\
Ir a la soda. Comprar & 8 & 2,72 \\
Jugar fútbol & 7 & 2,38 \\
Tener tiempo libre & 6 & 2,04 \\
Correr & 6 & 2,04 \\
Caminar & 6 & 2,04 \\
Estar afuera. Salir del aula & 6 & 1,70 \\
Ir al baño & 5 & 1,36 \\
Nada & 5 & 0,68 \\
Escuchar música. Jugar "play" & 294 \\
Ir a la biblioteca & 4 & 0,68 \\
Otros & 2 & 0,68 \\
No responde & 2,04 \\
No sé & 2 & 0 \\
NA & 2 & 100,00 \\
\hline Total de respuestas & 2 & \\
\hline & & 0 \\
\hline
\end{tabular}


URL: http://www.una.ac.cr/educare

CORREO: educare@una.cr

De acuerdo con Smaliroff (s. f.), el recreo es un "(...) intervalo entre las horas de clases escolares, es un momento en el cual los alumnos y los docentes se distienden de las actividades aúlicas, buscando un momento de vínculos espontáneos, recreación y relax" (p. 2). Precisamente lo que más les gusta a los niños y niñas del recreo, tiene que ver con actividades que permiten lo anterior: el esparcimiento, las relaciones con los otros y el desestrés. Un 42,17\% de las repuestas muestra que para los niños y niñas el juego resulta ser lo que más les gusta del recreo, tal como lo plantea Smaliroff (s. f. ) "(...) el juego es la principal actividad de las personas durante la infancia y una actividad necesaria y placentera durante toda la vida (p. 2).

Llama la atención que un porcentaje de las respuestas de niños y las niñas $(1,70 \%)$ hacen referencia a que lo que más les gusta del recreo es salir del aula, al argumentar "salir del aula", "estar afuera", "que salimos del aula", esto quizás se debe a que la mayor parte del tiempo pasan en un solo espacio físico y en algunos casos cerrado y sin adecuada ventilación e iluminación, por lo que salir del aula resulta ser el atractivo del recreo.

Con menores porcentajes se observan respuestas relacionadas con "ir al baño" $1,36 \%$, "escuchar música y jugar play" 0,68\% e "ir a la biblioteca" 0,68\%. Llama la atención que los niños y niñas prefieren jugar que ir al baño y además también les gusta tener la posibilidad de "escuchar música", "jugar con el play" y "leer en la biblioteca", actividades que, aunque no frecuentes entre la población participante, los sacan de la rutina escolar.

En la categoría de "otros" se encuentran respuestas como "pasar un tiempo sin la profe"," "pasear por las calles", "la cancha de fútbol", "los corredores", "compartir" y "al rato a llamar". Todo esto tiene que ver con las diversos intereses que tienen los niños y las niñas en los momentos de esparcimiento durante el período lectivo, los cuales se ven reflejados en los juegos que desarrollan.

Tabla 2

Juegos de los niños y las niñas durante el recreo

\begin{tabular}{lcc}
\hline Categoría & Frecuencia & $\%$ \\
\hline La anda & 85 & 29,51 \\
Congelado/ quemado & 40 & 13,88 \\
Ninguno/ nada & 21 & 7,29 \\
Fútbol / bola & 19 & 6,59 \\
Escondido & 17 & 5,90 \\
Policías y ladrones & 17 & 5,90 \\
Juegos tradicionales (suiza, elástico, trompo) & 15 & 5,20 \\
Atraparse/ perseguirse & 9 & 3,12 \\
Carreras/ correr / competencias & 9 & 3,12 \\
Caminar & 9 & 3,12 \\
Andar con mis amigos/ conversar/ compartir & 9 & 3,12 \\
\hline & & continúa
\end{tabular}




\begin{tabular}{lcc}
\hline Categoría & Frecuencia & $\%$ \\
\hline Cualquier cosa/ jugar & 7 & 2,43 \\
Toro & 6 & 2,08 \\
Molestar / Vacilar & 3 & 1,04 \\
Cueva & 2 & 0,69 \\
Otros & 15 & 5,20 \\
No sé & 1 & 0,34 \\
No aplica & 4 & 1,38 \\
\hline Total de respuestas & 288 & 100,00 \\
\hline
\end{tabular}

De la tabla 2, se desprende que todos estos juegos que los niños y niñas practican implican relacionarse y compartir con los demás y varían de acuerdo con la edad de los niños, por ejemplo los niños y niños de tercer grado prefieren jugar "la anda" y "congelado", mientras que los niños de sexto grado prefieren otras actividades que también tienen que ver con la relación que establecen con sus pares, en palabras de ellos "vacilo² con mis amigos", "yo no juego, yo camino en los pasillos", "caminamos por la escuela", "converso con mis amigas", "ando con mis amigos".

El rol docente resulta ser fundamental en todo lo relacionado con la jornada escolar, inclusive en lo relacionado con el tiempo de recreo. Por esta razón, se le preguntó a la población infantil entrevistada sobre lo que hacen sus maestros y maestras durante el recreo. A continuación el detalle.

Tabla 3

Lo que hacen los maestros y maestras durante el recreo

\begin{tabular}{lcc}
\hline Categoría & Frecuencia & $\%$ \\
\hline Comer. Ir a la soda & 55 & 22,35 \\
Cuidar a los niños. Cuidarnos. Vernos. & 55 & 22,35 \\
Descansar & 34 & 13,82 \\
Hablar con las otras maestras- Conversar. & 19 & 7,72 \\
Estar en la sala de profesores o el comedor & 13 & 5,28 \\
Revisar & 12 & 4,87 \\
Hacer sus cosas, sus trabajos. & 11 & 4,47 \\
Quedarse en el aula & 7 & 2,84 \\
\hline
\end{tabular}

continúa

2 En Costa Rica se utiliza esta palabra en sentido de molestar, gozar. 
URL: http://www.una.ac.cr/educare

CORREO: educare@una.cr

\begin{tabular}{lcc}
\hline Categoría & Frecuencia & $\%$ \\
\hline Ir a la dirección & 5 & 2,03 \\
Compartir con nosotros & 2 & 0,81 \\
No hacen nada. Vaguean & 2 & 0,81 \\
Regañan y aplican algunas medidas correctivas & 2 & 0,81 \\
Ir al baño & 2 & 0,81 \\
Jugar & 1 & 0,40 \\
Otros & 1 & 0,40 \\
No sé & 23 & 9,34 \\
No aplica & 1 & 0,40 \\
\hline Total de respuestas & 246 & 100,00 \\
\hline
\end{tabular}

De la tabla 3, se considera importante anotar que solo un $0,80 \%$ de las respuestas hace referencia al hecho de que los docentes comparten con los estudiantes en el momento de recreo. Como se puede ver, lo que principalmente hacen los docentes en este tiempo y de acuerdo con las opiniones de los niños y niñas, es "comer" $y$ "cuidar a los niños" ambas categorías con un porcentaje de $22,35 \%$.

El resto de porcentajes tienen que ver con actividades propias de los maestros y las maestras, y no tienen relación con los niños y niñas. En este sentido, se pueden mencionar las categorías de "Estar en la sala de profesores" con un 5,28\%, "Hablar con las otras maestras" con un $7,72 \%$ y "Revisar" con un $4,87 \%$.

Por otra parte, se evidencia que los porcentajes que hacen referencia a tener algún tipo de contacto con la población infantil está relacionado con el regaño, la censura, la vigilancia o las medidas correctivas, por ejemplo, en la categoría de "Regañan y aplican algunas medidas correctivas" $(0,81 \%)$ se evidencian respuestas como "nos regañan mucho" $y$ " nos quitan las botellas que juegan partido".

Si bien es cierto que los docentes tienen que velar por las seguridad de los niños en el momento de recreo, quizás sería mucho más significativo si buscaran estrategias de acercamiento maestro-estudiante para conocerles más de cerca, prevenir situaciones riesgosas y entre otros aspectos, propiciar un ambiente cálido, afectivo y significativo para sus estudiantes en estos momentos de esparcimiento. Precisamente como una manera de buscar estrategias para mejorar el recreo, se le consultó a los niños sobre lo que no les gusta del recreo, la tabla 4 refleja estas respuestas. 
Tabla 4

Lo que no le gusta a los niños y niñas del recreo

\begin{tabular}{lcc}
\hline Categoría & Frecuencia & $\%$ \\
\hline Que no dura tanto. Que es muy corto. Que se acaba. & 90 & 42,80 \\
No sentirme seguro, que me molesten, golpearme, lastimarme, que nos & & \\
golpean, me empujan & 40 & 19,04 \\
Que pelean. Las peleas & 20 & 9,52 \\
Que tiene algunas prohibiciones y vigilancias. & 9 & 4,28 \\
Todo me gusta & 7 & 3,33 \\
Que hay muchas personas & 4 & 1,90 \\
Que la soda se llena mucho & 3 & 1,42 \\
Factores relacionados con el cansancio & 3 & 1,42 \\
Los juegos bruscos. Las malas palabras & 2 & 0,95 \\
Otros & 7 & 3,32 \\
NA & 20 & 9,52 \\
No sé & 5 & 2,38 \\
\hline Total de respuestas & 210 & 100,00 \\
\hline
\end{tabular}

Según la tabla 4, a la mayoría de los entrevistados y entrevistadas, no le gusta el recreo porque el tiempo es muy corto, esto se evidencia con un $42,80 \%$ de las respuestas, algunas de las cuales son: "que dura poco", "que hay poco tiempo", "que no tenemos mucho tiempo" y"que se va rápido". Es importante anotar que este porcentaje corresponde tanto a niños y niñas de tercer y cuarto grado, como de quinto y sexto grado, es decir, indistintamente de la edad, la población escolar entrevistada considera que el tiempo que tienen los recreos no es suficiente $y$, por ende, manifiestan en primer lugar que lo que no le gusta del recreo es el poco tiempo que dura.

Los otros aspectos que no son del agrado de los niños y niñas con respecto al recreo tienen que ver con la inseguridad, las peleas, las prohibiciones, la cantidad de personas juntas en un mismo espacio y el cansancio.

Un $29,51 \%$ de las opiniones de los entrevistados refleja que lo que no les gusta del recreo se relaciona con los pleitos, las peleas, el sentirse amenazados o susceptibles de ser lastimados y los juegos bruscos. De esto se infiere que en el recreo ocurren situaciones que pueden atentar contra la seguridad de los niños y niñas, por tal motivo la supervisión de los adultos en este sentido, resulta fundamental. Al respecto, Aguilera (2010) manifiesta "(...) que la eduación debe revalorizar la importancia del recreo y de las actividades que en él se desarrollan (...)" (p. 3).

Por otra parte, un 4,28\% de las respuestas de la población infantil entrevistada, está centrado en que lo que no les gusta del recreo consiste en que tiene algunas prohibiciones y vigilancias, así, se destacan respuestas como "no jugar peleas", "que no podemos jugar brusco", "que Mister Vin no nos deja jugar" y "hacer fila". De este modo se evidencia que, a algunos 
URL: http://www.una.ac.cr/educare

CORREO: educare@una.cr

niños y niñas les gusta sentirse libres para hacer lo que quieren, incluso si se trata de jugar de peleas o jugar brusco. Una vez más se destaca la importancia de que los adultos asuman un rol importante en estos espacios de esparcimiento, y que indaguen y conozcan los gustos, intereses y preferencias de la población estudiantil.

Las categorías de "Que hay muchas personas" con un 1,90\% y "Que la soda se llena mucho" con un $1,42 \%$, se relacionan con la cantidad de personas que generalmente hay en los recreos, se infiere que a los niños y niñas que destacaron estos aspectos no les gusta la aglomeración de gente y, específicamente, cuando van a comprar a la soda, prefieren hacerlo cuando hay pocas personas. Esta última situación también puede ser analizada por las personas responsables y encargadas de la administración de los centros educativos. Es interesante que los mismos niños y niñas manifiestan que lo que no les gusta del recreo es todo aquello que implica cansarse, así en un 1,42\% de sus respuestas se argumenta que lo que no le gusta del recreo es "correr".

Además de lo anterior, como se puede ver en la tabla 4, un 3,33\% de las respuestas de los participantes muestra que todo les gusta del recreo. También un 3,32\% se ubican en la categoría de "Otros", en donde resaltan algunas respuestas como "estar sentado", "las escaleras", "quedarme sentada". Estos últimos aspectos tienen que ver con el aburrimiento y la infraestructura de la escuela, condiciones que quizás se convierten en limitantes para disfrutar del recreo. Además de lo anterior, otra limitante para disfrutar del recreo puede ser el tiempo que se le destina al mismo.

En la tabla 5 se sintetiza la información relacionada con la cantidad de recreos que le gustaría a los niños y niñas que hubiese.

Tabla 5

Cantidad de recreos que les gustaría a los niños y las niñas que hubiese

\begin{tabular}{lcc}
\hline Categoría & Frecuencia & $\%$ \\
\hline 5 recreos & 54 & 27,83 \\
4 recreos & 41 & 21,13 \\
6 recreos & 20 & 10,3 \\
Más de 10 recreos & 16 & 8,24 \\
3 recreos & 14 & 7,21 \\
10 recreos & 13 & 6,7 \\
7 recreos & 7 & 3,6 \\
8 recreos & 5 & 2,57 \\
9 recreos & 5 & 2,57 \\
Otros & 14 & 7,21 \\
NR & 1 & 0,51 \\
NA & 2 & 1,03 \\
No sé & 2 & 1,03 \\
\hline Total de respuestas & 194 & 100,00 \\
\hline
\end{tabular}


En la tabla 5 se refleja, en un 59,26\% de las repuestas, que los niños y las niñas preferirían tener entre 4 y 6 recreos, aspecto que permite deducir que los que tienen en la actualidad no están siendo suficientes para ellos. Por su parte en un 15,44\% se refleja que quieren de 7 a 10 recreos, también, en un $8,24 \%$ se desprende que quieren más de 10 recreos.

En la categoría de "Otros" con un 7,21\% de las respuestas, los niños y niñas entrevistadas argumentan que quieren "100,0000", "100,000000", "25 recreos", "todos los recreos del mundo", "625", "que cada hora hubiera uno"; respuestas que muestran lo mencionado anteriormente con respecto a la necesidad que evidencian los niños y niñas de tener estos espacios de recreo. Al respecto, Reyes (2005) argumenta que:

(...) Los estudiantes lo esperan con ansiedad probablemente como reacción a las clases aburridas o represivas. Sienten ese lapso como la oportunidad de ser libres, hasta de las normas esenciales de convivencia (p. 26).

En este sentido, es importante que el personal docente y las autoridades educativas analicen si la cantidad actual de recreos es lo suficientemente efectiva para lo que sus estudiantes requieren.

Ahora bien, en la tabla 6 se muestran las respuestas de la población infantil participante, con respecto a los lugares en los que juegan durante el recreo. Esto como otro aspecto importante de considerar en estos momentos de esparcimiento.

Tabla 6

¿Cómo describirías el lugar donde jugás en el recreo?

\begin{tabular}{lcc}
\hline Categoría & Frecuencia & $\%$ \\
\hline Hay corredores, gradas, barandas & 58 & 22,22 \\
Hay cosas naturales & 50 & 19,15 \\
Hay canchas, planché & 33 & 12,64 \\
Es lindo y espacioso & 18 & 6,89 \\
Es un patio. Hay una plaza & 16 & 6,13 \\
Hay cemento & 14 & 5,36 \\
No es lindo. Es desordenado. & 7 & 2,68 \\
Juego en el aula & 6 & 2,29 \\
Es un lugar divertido & 5 & 1,91 \\
\hline
\end{tabular}


URL: http://www.una.ac.cr/educare

CORREO: educare@una.cr

\begin{tabular}{lcc}
\hline Categoría & Frecuencia & $\%$ \\
\hline Es la biblioteca & 3 & 1,14 \\
Es pequeño & 1 & 0,38 \\
Otros & 21 & 8,04 \\
NR & 9 & 3,44 \\
NA & 15 & 5,74 \\
No sé & 5 & 1,91 \\
\hline Total de respuestas & 261 & 100,00 \\
\hline
\end{tabular}

Un 65,5\% de las respuestas de los niños y niñas se centran en describir los espacios de recreo con respecto a la infraestructura y a sus características generales. De este modo, se pueden mencionar respuestas como "hay zacate", "una barandita", "tiene tubos, tiene pilas, pasadizos, aulas", "es de cemento", y entre otras "es una cancha".

Por otra parte, un 6,89\% de las respuestas centran sus opiniones en clasificar el espacio del recreo como "lindo o espacioso" al destacar "es un poco grande y hay bastante espacio", "porque hay más espacio y es más lindo". En contraposición, un 2,68\% de las respuestas a la entrevista, se agrupa en la categoría de "No es lindo. Es desordenado". Ambas categorías reflejan que hay diversidad de espacios de recreo en los centros educativos y, como bien se puede apreciar, el porcentaje de niños y niñas que se sienten a gusto en estos espacios, es mayor que el porcentaje de los que está a disgusto en el espacio.

Se desprende, entonces, que el espacio físico y de infraestructura con que los niños y niñas cuentan durante el recreo, también incide en su bienestar y satisfacción manifestado durante este período de la jornada escolar. Al respecto, Williams, citado por Pavía (2005), destaca la relación entre el fenómeno educativo y el espacio físico al plantear de este último que:

(...) su diseño, disposición, interés, limpieza y seguridad, su capacidad para exaltar o relajar (...) todo va a impactar en la experiencia recreativa, simplemente porque todas las formas de recreación al aire libre dependen de la interacción de individuos (grupos) y el medio ambiente. (p. 45)

Sumado a lo anterior, la National Association for Sport and Physical Education (2006) recomienda que las escuelas deben proporcionar a los estudiantes el acceso a espacios seguros, equipo adecuado y la supervisión de adultos calificados, para que estos puedan gozar de las condiciones adecuadas durante el tiempo de recreo. 
Es así como queda en evidencia que durante el tiempo de recreo los niños y niñas deben estar acompañados por personas adultas, con el fin de que velen por su seguridad e integridad. En la tabla 7 se reflejan las respuestas del estudiantado a la pregunta: “Quiénes cuidan a los niños y las niñas durante el recreo?"

Tabla 7

Personas que cuidan a los niños y niñas durante el recreo

\begin{tabular}{lcc}
\hline Categoría & Frecuencia & $\%$ \\
\hline Los maestros & 131 & 55,50 \\
Nadie & 42 & 17,79 \\
El guarda & 22 & 9,32 \\
Director o directora & 17 & 7,20 \\
El conserje & 12 & 5,08 \\
Otros & 4 & 1,69 \\
NR & 1 & 0,42 \\
NA & 3 & 1,27 \\
No sé & 4 & 1,69 \\
\hline Total de respuestas & 236 & 100,00 \\
\hline
\end{tabular}

Las respuestas de la población infantil reflejan, en su mayoría -con un 55,50\%-que los docentes son los encargados del cuido durante el recreo. Es alarmante la segunda categoría, en la que se aprecia que según un $17,79 \%$ de las respuestas, nadie cuida a los niños y niñas durante el recreo. En este sentido, Jarret y Waite-Stupiansky (2009) argumentan que, en los patios de la escuela, es necesario contemplar las necesidades de supervisión por parte de los adultos. Por lo tanto, resulta preocupante que algunos entrevistados mencionen que nadie los cuida durante el recreo.

Ahora bien, un $21,6 \%$ de las respuestas destacan que otras de las personas que se encargan de cuidar a los niños y niñas durante el recreo son los guardas, directores y conserjes. Finalmente, un 1,69\% de las respuestas, señala, en la categoría de "Otros", diferentes personas que los cuidan durante el recreo: "la de la biblioteca", "mamá", "los grandes defienden a los pequeños" $y$ "los grandes".

De estas respuestas se desprende que la responsabilidad del cuido durante este momento de la jornada diaria se asigna a diferentes personas, quienes son parte del ámbito educativo, en este caso la bibliotecaria o inclusive los padres y madres de familia. Por otra parte, resulta 
URL: http://www.una.ac.cr/educare

CORREO: educare@una.cr

interesante ver cómo los niños y niñas evidencian esta responsabilidad en sus mismos pares, en el sentido de que los grandes cuidan a los pequeños, lo cual muestra un comportamiento de solidaridad y apoyo entre compañeros.

\section{Conclusiones}

De la investigación realizada se desprende que el tiempo de recreo resulta ser una necesidad fundamental para los niños y niñas dentro del proceso educativo. Por tanto, no puede ser considerado como un aspecto poco importante o al que no hay que prestarle la debida atención, pues como se vio, el recreo permite que los estudiantes puedan descansar, hacer sus necesidades básicas, alimentarse, compartir con sus compañeros y compañeras, jugar y desestresarse de la rutina académica.

Por otra parte, resulta importante señalar que el juego, en sus diferentes manifestaciones, es una característica fundamental del recreo, según lo destacan los niños y las niñas. Independientemente del grado en el que se encuentren los entrevistados y las entrevistadas, manifiestan, en su mayoría, que el juego es lo que más les gusta del recreo, es lo que prefieren hacer durante el recreo y es lo más importante del recreo. De este modo, se considera necesario analizar: ¿Cuáles son las implicaciones del juego en el recreo? ¿De qué manera los adultos podrían aprovechar estos momentos para propiciar espacios formativos en ellos? Asimismo, es importante reflexionar sobre: ¿Cuáles son los tipos de juegos que están jugando los niños y niñas? ¿Cuáles juegos les gusta jugar? ¿Cómo responder a los intereses y necesidades que tienen los niños y niñas en función del juego durante el recreo?

Precisamente, los cuestionamientos anteriores develan la responsabilidad que deben asumir los adultos en las instituciones educativas con respecto a los recreos. Así, se desprende que las obligaciones de los adultos no solo deben girar en torno a propiciar espacios seguros, supervisados y libres de peligros para los niños y las niñas, sino que también buscar diversas opciones y propuestas que les permitan disfrutar del recreo de una mejor forma. En este sentido, cabe señalar que el hecho de que los adultos jueguen y compartan con los niños y niñas durante el tiempo de recreo, resulta ser un aspecto significativo e importante para ellos.

La mayoría de las respuestas refleja que lo que no les gusta a los niños y niñas del recreo es el corto tiempo que tienen y lo pequeño que les resulta para realizar sus actividades. A esto se puede agregar que otro aspecto de vital importancia en el recreo tiene que ver con los espacios en los que niños y niñas disfrutan de este momento. Estos espacios deben ser propicios y aptos para jugar, seguros, techados, amplios, con zonas verdes y con zonas de juegos. Todo esto permitirá un mejor desenvolvimiento de los niños y niñas durante el tiempo de recreo.

Este análisis en torno a la temática de los recreos escolares constituye un insumo importante para generar mejoras en los procesos educativos de manera integral y para responder a las características, intereses y necesidades de los niños y niñas escolares. 


\section{Recomendaciones}

A la luz de las opiniones del estudiantado entrevistado, se plantean algunas recomendaciones fundamentales para las entidades educativas responsables y personas encargadas de la dinámica escolar.

1. Revisar los tiempos y cantidad de recreos en la escuela pública costarricense, de manera que se tome en cuenta que los niños y niñas requieren de estos períodos para poder mejorar en sus procesos de atención y concentración durante las clases. Analizar la efectividad de plantear únicamente tres recreos con tiempos entre 10 y 20 minutos durante la jornada escolar.

2. Valorar los espacios en donde los niños y niñas se desenvuelven y comparten durante el recreo. Estos espacios deben ser seguros y aptos para el desarrollo de actividades grupales, al aire libre y, en general, brindar oportunidad para que los niños y niñas se desenvuelvan de la mejor manera en el tiempo de recreo.

3. En relación con lo anterior, es necesario que además de que existan espacios físicos (de infraestructura) adecuados para que los niños y niñas disfruten durante el recreo, resulta imprescindible también que la población escolar tenga los materiales y juegos que les permitan realizar las actividades que les gustan, por ejemplo, los materiales que propicien el desarrollo de la motricidad gruesa y el movimiento.

4. Es necesario indagar, con los niños y niñas de la escuela, ¿qué es lo que les gusta hacer durante el recreo?, y de esta manera intentar responder a estos intereses. Así, se pueden implementar diferentes actividades y juegos durante el recreo: juegos tradicionales, juegos de mesa, juegos de movimiento, rondas, entre otros. Por esto es fundamental abrir espacios para escuchar a la población escolar y para que el docente se convierta en un investigador de su propia realidad educativa.

5. Resulta necesario abordar el rol de las personas adultas, de docentes, administradores educativos y demás personal de las comunidades educativas, como guardas, conserjes, secretarias, entre otros. Como bien se vio en las respuestas de los niños y las niñas, los adultos encargados, principalmente personal docente, tienen un papel de supervisar y vigilar los recreos. A la luz de esta situación, lo que se propone es que esta función trascienda y los docentes puedan tener un mayor acercamiento hacia sus estudiantes compartiendo, disfrutando y jugando con ellos.

6. En relación con lo anterior, es importante que el grupo de docentes busque espacios de diálogo en los que aborde el tema de los recreos en la escuela, con el propósito de que pueda indagar sobre las necesidades, intereses y solicitudes de la población infantil con respecto al recreo. Estos espacios de diálogo pueden propiciar la construcción conjunta de estrategias que les permitan a los niños y niñas disfrutar del recreo, es decir, los mismos niños y niñas son los que pueden proponer diferentes actividades para realizar en el recreo con la guía y acompañamiento de las personas adultas. 
URL: http://www.una.ac.cr/educare

CORREO: educare@una.cr

7. Otra de las recomendaciones que se plantea gira en torno al argumento de Jarret y WaiteStupiansky (2009), quienes argumentan que al considerarse el recreo como un derecho y no como un privilegio, no es adecuado imponer como castigo su eliminación. Como bien se ha hablado anteriormente, el recreo resulta ser un beneficio para la atención de los niños, para mantener el interés de las actividades académicas y para mejorar el rendimiento escolar. A la luz de estos argumentos, no es adecuado privar a los niños y niñas de este espacio tan necesario para ellos dentro de la jornada escolar.

8. Además de lo anterior, se plantea la idea de crear junto con los estudiantes y las estudiantes un recreo formativo, entendido como un espacio en el que los niños y niñas van a disfrutar de su tiempo libre y en donde van a tener la oportunidad de aprender y vivenciar algunos aspectos importantes: reglas para estar en el recreo, socializar y entablar relaciones interpersonales, aprendizajes necesarios para la convivencia con los demás y consigo mismos, criticidad y reflexión sobre sus propias actuaciones y las de los demás.

De este modo, un recreo formativo abre paso a que sean los mismos niños y niñas los que den respuesta a la interrogante: ¿Qué aprendemos en el recreo?". Si se propician espacios de diálogo, reflexión y creación de estrategias relacionadas con los recreos, estos serán más significativos y pertinentes para los niños y niñas y, además, se convertirán en otra oportunidad de recrearse y formarse al mismo tiempo.

Los desafíos en torno a la temática son bastantes y con este artículo se pretende hacer una invitación a los docentes y encargados para buscar espacios de diálogo y reflexión con sus estudiantes y con sus colegas, para ofrecer mejores espacios y tiempos de recreo a sus estudiantes. De esta manera, se estará respondiendo a las características, intereses y necesidades de los niños y niñas en edad escolar y, a la vez, se contribuye con la calidad de los procesos educativos.

\section{Referencias}

Aguilera, E. (2010). ¡Recreo!... ¡Vamos a jugar! Espacios y juegos: Una responsabilidad compartida. Recuperado de http://www.colaborando.edu.ar/PcWeb/Contenido/Actividad44/File/ Recreo,\%20vamos\%20a\%20jugar.pdf

Álvarez, F., Muñoz, F., Martínez, G., Trujillo, J. A., Echeverry, J. L. y Salazar, D. M. (2010). Proyecto de lúdica, Tiempo libre y recreación. Colombia: Envigado. Recuperado de http://mua. edu.co/mua/images/Archivos/docentes/edfisica/proyecto\%20de\%20ludica $\% 20$ \%20 tiempo\%20libre.pdf

Código de la niñez y la adolescencia (6 de febrero de 1998). La Gaceta № 26. Recuprado de http://www.tse.go.cr/pdf/normativa/codigodelaninez.pdf

Convención sobre los Derechos del Niño. (2005). Lima, Perú: Save the Children.

Hernández, R., Fernández, C. y Baptista, P. (2010). Metodología de la investigación (5ª ed.). Perú: McGraw-Hill. 
Jarret, O. (2002). El recreo en la escuela primaria: ¿Qué indica la investigación? (Recess in Elementary School: What Does the Research Say?). Champaing, IL: Eric Clearinghouse on Elementary and Early Childhood Education. De la base de datos Eric Digests (ED467567). Recuperado de_http://www.eric.ed.gov/PDFS/ED467567.pdfh

Jarret, O. y Waite-Stupiansky, S. (2009). Recess-It's Indispensable! [El recreo es imprescindible]. [Play, Policy, and Practice Interest Forum, NAEYC]. Recuperado de http://www.naeyc.org/ files/yc/file/200909/On Our Minds 909.pdf

National Association for Sport and Physical Education. (2006). Recess for Elementary School Students [Recreo para los estudiantes de la escuela primaria]. Recuperado de http://www. aahperd.org/naspe/standards/upload/Recess-for-Elementary-School-Students-2006.pdf

Pavía, V. (2005). El patio escolar: El juego en libertad controlada. Un lugar emblemático territorio de pluralidad. Buenos Aires: Centro de Publicaciones Educativas y Material Didáctico. Recuperado de http://books.google.co.cr/books?id=20hl47iJjgcC\&printsec=frontcover\& source=gbs_ge_summary_r\&cad=0 - v=onepage \&q\&f=false

Pérez, L. y Collazos, T. (2007). Los patios de recreo como espacios para el aprendizaje en las instituciones educativas. Sedes Pablo Sexto en el Municipio de Dosquebradas. Universidad Tecnológica de Pereira. Colombia. Recuperado de http://repositorio.utp.edu.co/dspace/ bitstream/11059/761/1/371621P438lp.pdf

Pica, R. (s. f.). 7 Reasons why Kids Need Recess [7 razones del porqué los niños necesitan el recreo]. Recuperado de http://www.selfgrowth.com/articles/7_Reasons_Why_Kids_Need_Recess.html

Reyes, J. I. (2005). La agresividad y violencia en los juegos de los niños durante el recreo escolar. Recuperado de http://www.ucol.mx/egeneros/admin/archivos/agresividad_violencia_ juegos.pdf

Smaliroff, C. M. (s. f.). Juegotecas para los recreos escolares. Los recreos. Aprender y compartir. Escuela Nueva Juan Mantovani. Recuperado de http://www.preveninos.com/Files_ Proyectos/Proy_256.pdf

Vicente, R. y Castro, M. (2010). Promoviendo los derechos de niños, niñas y adolescentes: Conozcamos las leyes. Heredia, Costa Rica: Instituto de Estudios Interdisciplinarios de la Niñez y la Adolescencia. Universidad Nacional.

Cómo citar este artículo en APA:

Chaves, A. L. (2013). Una mirada a los recreos escolares: El sentir y pensar de los niños y niñas. Revista Electrónica Educare, 17(1), 67-87. Recuperado de http://www.revistas.una. ac.cr/index.php/EDUCARE/issue/current

Nota: Para citar este artículo en otros sistemas puede consultar el hipervínculo "Como citar el artículo" en la barra derecha de nuestro sitio web:

http://www.revistas.una.ac.cr/index.php/EDUCARE/index 